\title{
Review of Pushing the Margins: Women of Color and Intersectionality in LIS
}

Maisha Carey

University of Delaware

Abstract: Review of Chou, Rose L., \& Pho, Annie. (Eds). (2018). Pushing the Margins: Women of Color

and Intersectionality in LIS. Sacramento, CA: Library Juice Press. 488 p.

Keywords: equity, diversity, inclusion, race, gender, intersectionality

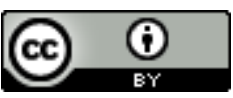

This is an Open Access article distributed under the terms of the Creative Commons Attribution 4.0 International License (http://creativecommons.org/licenses/by/4.0), which permits unrestricted use, distribution, and reproduction in any medium, provided the original work is properly cited. 
It seems that Equity, Diversity and Inclusion (ED\&l) are on everyone's agenda these days. The American Library Association has recently adopted ED\&I as its fourth strategic direction and thankfully, many Library and Information Science (LIS) institutions are making an attempt to "do the right thing" through trainings, values statements, initiatives and committees. Among their goals is a focus on staff recruitment and retention, a task that can be challenging in a field that is over $80 \%$ white but overwhelmingly sees itself as progressive and welcoming. The current focus on staff diversity allows our institutions to present a concise solution (increasing demographic diversity) to what is a complex and multifaceted dilemma (institutional racism and oppression). Scholars are pushing our institutions to consider that dilemma through conversations on a range of topics including neutrality, social justice, white fragility, allyship, and vocational awe - all topics that force us as individuals, organizations, and as a professional community to reexamine our power, privilege and biases.

Entering into and providing new perspectives in this evolving conversation about ED\&l is Pushing the Margins: Women of Color and Intersectionality in LIS, edited by Rose L. Chou and Annie Pho. This curated collection of 17 essays, studies, and interviews uses intersectionality as a framework to explore the unique and varied experiences of women of color in LIS. Intersectionality is a concept given language by law professor and scholar Kimberlé Crenshaw in 1989 and used to describe how the discrimination and oppression faced by those with multiple marginalized identities are relational and cannot be fully understood without considering how they intersect and thus transform in the lives of individuals. Pushing the Margins is the third book in their Series on Critical Race Studies and Multiculturalism in LIS, published by Litwin Books and Library Juice Press. The book, as well as the series as a whole, aims to expand the conversation around issues of ED\&l in library and information science and make space for more nuanced and specific explorations. In their introduction Chou and 
Pho state their intentions: to shift how we think of diversity by creating space for the varied voices and experiences of women of color in LIS, to counter the misappropriation of intersectionality and return women of color to its center, and to raise consciousness by building community. Chou and Pho provide a list of texts from outside of LIS that have influenced their work: Black Feminist Thought, Women, Race + Class, Sister Outsider, The Combahee River Collective Statement and This Bridge Called My Back. This book, in the tradition of these salient works, does not aim to be a comprehensive account of what it means to be a woman of color in librarianship. Instead, it invites a space for shared theorizing among each chapter's authors and the readers that engage with these ideas and take up the questions and challenges posed within.

In the foreword, librarian and scholar Fobazi Ettarh asserts the centrality of women of color in the concept of intersectionality amidst attempts to co-opt, soften or even silence the conversation around marginalization. Ettarh provides an in-depth definition of the many facets of intersectionality exploring structural, political and cultural intersectionality with relevant and familiar examples. With this deeper understanding of intersectionality, Ettarh hopes that we can strengthen and articulate the need for deeper and more complex ED\&l efforts. Ettarh's message makes me wonder if with intersectionality we could have conversations and support efforts that go beyond increased demographic representation, giving voice to the complex interplay of personal identity and the systems of oppression we inadvertently create and uphold.

The remaining chapters in this volume represent a variety of approaches to examining how women of color have and continue to experience and influence the field of librarianship and LIS in general. Like friends gathering at a kitchen table, the authors bring their unique interests, experiences and questions to a conversation about being and supporting women of color in LIS. The chapters do 
not follow a formulaic structure, but rather there is a natural flow between the concepts with the collection framed by explorations of the past and the future. The first chapter by Caitlin M.J. Pollock and Shelly P. Haley explores how Black women librarians have historically navigated, resisted, and made use of the racial and gender norms of librarianship. In the last chapter, authors Todd Honma and Clara M. Chu engage in a critical dialogue to consider how we can strengthen and expand the ways we interrogate our field. In between there are personal and research-based accounts on community building and support, invisible and devalued labor and examples of how identity informs professional practice. While race and gender are foregrounded in this volume on women of color, some of the authors address other facets of their identity that impact how they experience LIS, including age, sexual orientation, religion and ethnicity. Many of the chapters use the racial demographics of LIS and academia along with experiences of being "the only" to frame the need for this conversation in the making.

DuBoisian notions of double consciousness (1903) show up frequently, if not always noted, in Pushing the Margins. This sense of seeing oneself through the lens of outsiders is essential to what this collection has to teach us about the additional energy women of color have to expend in making sense of and navigating the expectations placed upon them by their colleagues and the profession as a whole while remaining connected to their individual and social identities. This consciousness is only heightened when navigating largely white professional spaces where women of color may be invisible and hypervisible, as described adeptly by authors Jennifer Brown and Sophia Leung in their chapter.

Pressure to both seamlessly fit in and serve as an outspoken representative for those on the margins can be taxing and deter women from taking on the risk associated with articulating or challenging what is unfairly expected of them. When faced with this kind of pressure to stay quiet or 
speak up, along with the self-consciousness that comes with being tokenized, why would women of color in LIS still do diversity work? Brown and Leung say they do it to be of service to their community and because the silence and complacency that would take the place of this work is simply intolerable. In their study on identity activism, and self-care authors Alanna Aiko Moor and Jan Estrellado reported that participants participated in activism because they were raised to, as a reaction to their own experiences of oppression, or for fear that no one else would. If silence or inaction are unacceptable, then the only option is to speak up, take action, and navigate the consequences.

By contributing to Pushing the Margins, the authors in this volume are all taking part in an act of service. In telling their stories and examining the experiences of others they turn a mirror to our professional community and ask us to consider what role we play in either pushing against or upholding the status quo. For readers looking for practical solutions and next steps some of the authors offer suggestions related to hiring, outreach, accountability and support. But if you plan to thumb through looking for those action steps, you would miss out on engaging with these voicedperspectives and the chance to interrogate the real and systemic issues that have shaped the experiences of those centered here and our profession. The editors intentionally rooted this volume in the feminist technique of consciousness raising which makes space for women to share their stories and build community where they would once have felt isolated by their experiences of marginalization. This volume succeeds in inviting the reader into that space, to listen and learn from the experiences of the authors, lend their voices to the conversation, and seek out what they have yet to learn.

As the relational, non-linear idea of intersectionality so perfectly represents, there is no straight line between problem and solution as it relates to the marginalization faced by women of color and other traditionally underrepresented groups in LIS, and we should not seek one. Instead, we must 
continue to pull up to the kitchen table and continue this conversation, listening to and legitimizing one another's voices and exploring how intersectionality helps make sense of the challenges we face. Part of that conversation will indelibly be a critique of our professional community and how it seeks to correct those challenges yet reinforces the very structures that make them possible. It is not an easy conversation to have, but one that we must continue if we truly want to do the right thing. 